\title{
Stability Analysis of Multiple State-based Schedulers with CSMA
}

\author{
Chithrupa Ramesh, Henrik Sandberg, and Karl Henrik Johansson
}

\begin{abstract}
In this paper, we identify sufficient conditions for Lyapunov Mean Square Stability (LMSS) of a contention-based network of first-order systems, with state-based schedulers. The stability analysis helps us to choose policies for adapting the scheduler threshold to the delay from the network and scheduler. We show that three scheduling laws can result in LMSS: constant-probability laws and additively increasing or decreasing probability laws. Our results counter the notions that increasing probability scheduling laws alone can guarantee stability of the closed-loop system, or that decreasing probability scheduling laws are required to mitigate congestion in the network.
\end{abstract}

\section{INTRODUCTION}

Event-based systems have been proposed as a means to reduce congestion in Networked Control Systems (NCS) [1], [2]. Multiple closed-loop systems that share a communication channel between their respective sensors and controllers, as shown in Fig. 1, could use event-triggers to adapt their transmission rates to the traffic in the network. This brings benefits to the entire network, as each closed-loop system loses fewer packets due to congestion. However, in such a network, the event arrival times cannot be anticipated and scheduled. Thus, a Contention Resolution Mechanism $(\mathrm{CRM})$ is required to negotiate channel access between multiple event-packets from contending loops. The stability and performance of the resulting network of closed-loop systems is difficult to analyze. Consequently, design principles for event-based systems that guarantee stability, in a stochastic sense, are not easily obtained.

Event triggers are designed to detect a level crossing. However, how should these levels be chosen, especially after a packet failure? When sufficient packets do not reach the controller, either due to physical losses in the medium or insufficient triggering of the state, decreasing the levels is required to attain stability, in a stochastic sense. However, packet failures could also be due to collisions, while using the CRM, in which case increasing the level might be seen as necessary to alleviate congestion in the network. This is the same principle used in congestion control in TCP/IP or in the backoff mechanism in Carrier-Sense Multiple Access (CSMA) protocols. However, there is no guarantee that this policy leads to stability of the closed-loop system. What does this mean for the design of event-based systems? How should

This work was supported by the Swedish Research Council, VINNOVA (The Swedish Governmental Agency for Innovation Systems), the Swedish Foundation for Strategic Research, the Knut and Alice Wallenberg Foundation and the EU projects FeedNetBack and Hycon2.

C. Ramesh, H. Sandberg and K.H. Johansson are with the ACCESS Linnaeus Centre, Electrical Engineering, KTH Royal Institute of Technology, Stockholm, Sweden. $\{$ cramesh, hsan, kallej\}@ee.kth.se

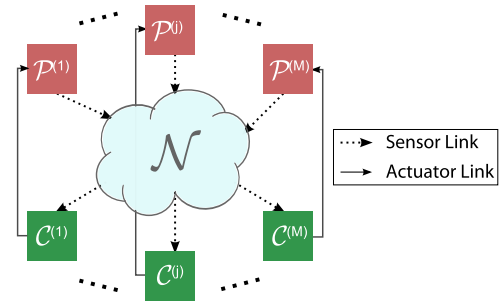

Fig. 1. $\quad M$ closed-loops, consisting of a plant $(\mathcal{P})$ and a controller $(\mathcal{C})$ each, use a shared network $(\mathcal{N})$ for communication between the respective sensors and controllers. Note that the controllers and actuators communicate over a dedicated point to point link, which suffers no losses.

the levels be selected, to ensure stability of the network, the lack of which results in congestion, and stability of the closed-loop system?

We consider a network of closed-loop systems that use a state-based scheduler, with $p$-persistent CSMA [3] as the CRM, as shown in Fig. 2. The state-based scheduler is a local selection mechanism at each node, which thresholds a function of the state to determine if a packet must be selected for transmission. The threshold itself is a function of the delay, or the elapsed time since the last transmission, to adapt transmission rates to the plant state and network traffic. Our goal is to identify stability conditions for a network of such systems.

The main contribution of this paper is an analysis of Lyapunov Mean Square Stability (LMSS) in this setup. We find that LMSS is achievable, if the probability of increasing delay is suitably restricted. Any appropriate selection of levels, following a non-transmission, must be tailored to achieve this property. The explicit relationship between a given level selection mechanism and the probability of delay involves other factors, such as the congestion in the network and the dynamics of the plant itself. Thus, it is not possible to claim that a certain level selection mechanism will always result in stability or instability of the system.

A second contribution is the proposal of three scheduling laws, based on the above stability analysis. Our results provide sufficient conditions for LMSS for these laws. The scheduling probabilities are mandated to remain constant, or increase or decrease additively, with increasing delay, according to these laws. Such variations in the scheduling probability can be effected by varying the scheduler threshold (level), specific to network conditions and system dynamics.

The problem of level selection after a packet loss was introduced in [4], where the authors evaluated the control cost of level triggering subject to i.i.d packet losses. Stochastic 


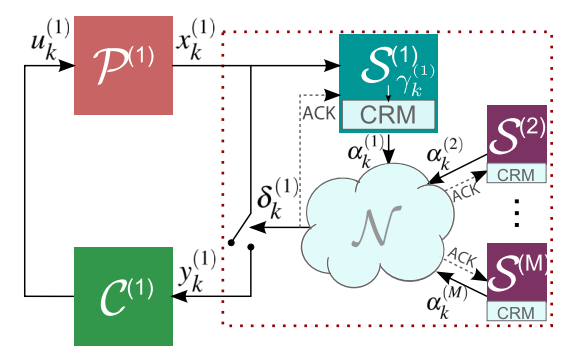

Fig. 2. An overview of a multiple access network $(\mathcal{N})$ of plants $\left(\mathcal{P}^{(j)}\right)$, state-based schedulers $\left(\mathcal{S}^{(j)}\right)$ and controllers $\left(\mathcal{C}^{(j)}\right)$, for $j \in\{1, \ldots, M\}$, with an explicit CRM in the MAC.

stability of event-based systems with i.i.d intervals between arrivals have been studied in [5], [6]. However, event arrivals in a contention-based network are not i.i.d [7], and the event arrivals considered in this paper exhibit a dependence on the delay since the last transmission. The design of event-based systems in a contention-based network has been dealt with in [8], but without modelling collisions in such networks. The notion of stability that we use in this analysis has been used in [9], to analyze i.i.d erasures, with a provision to extend to Markov models, in NCSs.

The rest of this paper is organized as follows. The problem formulation, along with a Markov chain representation, is presented in Section II. The main results on sufficient conditions for LMSS are presented in Section III, and three scheduling laws are presented in Section IV. Some examples and conclusions follow in Sections V and VI, respectively.

\section{Problem Formulation}

We consider a network of $M$ identical event-based systems, shown in Fig. 2. We first describe a model for each closed-loop system in the network, and then present a model for the interaction of the $M$ systems in the network.

\section{A. Closed-loop System Model}

The network on the sensor link can be modelled from the perspective of a single closed-loop system, as illustrated in Fig. 3. We describe each block in this model below.

Plant: The plant $\mathcal{P}$ has state dynamics given by

$$
x_{k+1}=a x_{k}+b u_{k}+w_{k},
$$

where $a, b \in \mathbb{R}, w_{k}$ is i.i.d. zero-mean Gaussian with variance $\sigma_{w}^{2}$, and $x_{0}$ has a zero-mean bounded distribution. Scheduler: A local scheduler $\mathcal{S}$ is situated in the sensor node, and decides if the state is an event, to be scheduled for transmission, or not. The scheduler output is denoted $\gamma_{k} \in$ $\{0,1\}$, and $\gamma_{k}=1$ in the case of an event. The innovations in the state, with respect to the observer $(\mathcal{O})$ across the network, determine the scheduler output $\gamma_{k}$, as given by

$$
\gamma_{k}= \begin{cases}1, & \left|x_{k}-\hat{x}_{k \mid \tau_{k-1}}^{s}\right|^{2}>\epsilon_{d}, \\ 0, & \text { otherwise. }\end{cases}
$$

Here, $\hat{x}_{k \mid \tau_{k-1}}^{s}=a \hat{x}_{k-1 \mid k-1}^{c}+b u_{k-1}, \hat{x}_{k-1 \mid k-1}^{c}$ denotes the estimate at the controller, defined in (5) below, and $\tau_{k}$ is the time index of the last received packet, given by $\tau_{k}=$

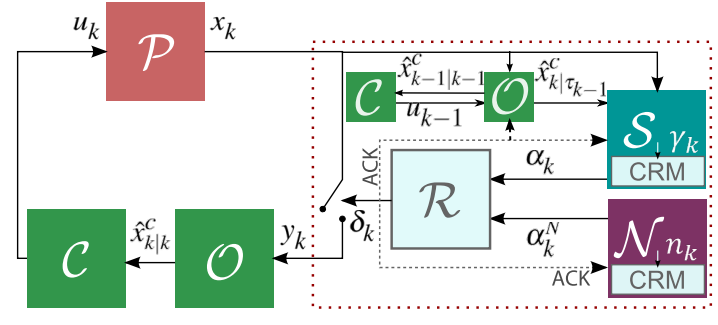

Fig. 3. A model of the closed-loop system from the perspective of a single NCS in the network. The other control loops in the network are abstracted by the network traffic block $(\mathcal{N})$. The resolution block $(\mathcal{R})$ maps the CRM output $\alpha$ to the MAC output $\delta$. A copy of the observer $(\mathcal{O})$ and controller $(\mathcal{C})$ are required at the scheduler for this architecture to be feasible.

$\left\{\max \{n,-1\}: \delta_{n}=1, n \leq k\right\}$. Also, $\epsilon_{d}>0$ is the scheduler threshold, and it may vary with the delay, i.e., $\epsilon_{d}$ need not be equal to $\epsilon_{d-1}$. Here, $d_{k}=k-\tau_{k}$ is the delay since the last received packet at time $k$. The estimate $\hat{x}_{k \mid \tau_{k-1}}^{s}$ is equal to the estimate at the controller $\hat{x}_{k \mid k}^{c}$, if the current packet is not scheduled for transmission. To realize such a scheduling policy, the observer and controller must be replicated within the scheduler, and an explicit acknowledgement (ACK) of a successful transmission is required.

Network: The network $\mathcal{N}$ generates exogenous traffic, as is indicated by $n_{k} \in\{0,1\}$. It takes a value 1 when a network source generates an event, and 0 otherwise. The network traffic is also stochastic, and hence, $n_{k}$ is a binary random variable, which is not required to be i.i.d.

CRM: The CRM resolves contention between simultaneous channel access requests. For simplicity, we assume that the network uses $p$-persistent CSMA with no retransmissions, in this paper. The output of the CRM is denoted $\alpha_{k} \in\{0,1\}$, and we have

$$
\mathbb{P}\left(\alpha_{k}=1 \mid \gamma_{k}=1\right)=p_{\alpha}
$$

where, $p_{\alpha}$ is the persistence probability of the CRM. Thus, with probability $\bar{p}_{\alpha}=1-p_{\alpha}$, some events are suppressed by the CRM and not permitted to access the medium. Similarly, $\alpha_{k}^{N}$ is the CRM output for the rest of the network, and $\mathbb{P}\left(\alpha_{k}^{N}=1 \mid n_{k}=1\right)=p_{\alpha}$.

The resolution block $(\mathcal{R})$ maps the transmission attempts $\alpha_{k}$ and $\alpha_{k}^{N}$ to the MAC output $\delta_{k}$, as given by

$$
\delta_{k}=\alpha_{k}\left(1-\alpha_{k}^{N}\right)
$$

where, $\left(\delta_{k}=1\right)$ indicates that a successful transmission of the event has occurred. This is possible only when the CRM permits a transmission and none of the other nodes attempt to transmit.

Observer $(\mathcal{O})$ : The input to the observer is the received measurement signal $y_{k}=\delta_{k} x_{k}$. The observer generates the estimate $\hat{x}_{k \mid k}^{c}$ as given by

$$
\hat{x}_{k \mid k}^{c}=\bar{\delta}_{k}\left(a \hat{x}_{k-1 \mid k-1}^{c}+b u_{k-1}\right)+\delta_{k} x_{k},
$$

where, $\bar{\delta}_{k}=1-\delta_{k}$ takes a value 1 when the packet is not transmitted. In such a case, the estimate is given by a modelbased prediction from the last received data packet at time 
$\tau_{k}$. The estimation error is defined as $\tilde{x}_{k \mid k}^{c} \triangleq x_{k}-\hat{x}_{k \mid k}^{c}$, and $P_{k \mid k}=\mathbb{E}\left[\left|\tilde{x}_{k \mid k}^{c}\right|^{2}\right]$ is the estimation error covariance.

Controller $(\mathcal{C})$ : The controller generates the signal $u_{k}$ based on the estimate alone, as given by

$$
u_{k}=-L \hat{x}_{k \mid k}^{c}
$$

where $L$ is the controller gain, and is chosen to optimize a control cost, such as an infinite horizon Linear Quadratic Gaussian (LQG) cost function.

We are interested in investigating mean square boundedness of the state in steady state, or LMSS, sometimes used without the epithet 'Lyapunov', as defined below.

Definition 2.1 (Lyapunov Mean Square Stability [10]):

The state is said to possess mean square stability if given $\zeta>0$, there exists $\xi(\zeta)>0$ such that $\left|x_{0}\right|<\xi$ implies

$$
\limsup _{k \rightarrow \infty} \mathbb{E}\left[\left|x_{k}\right|^{2}\right] \leq \zeta
$$

The Certainty Equivalence Principle has been shown to hold in the architecture described in (1)-(6) in [11]. Thus, we can translate the LMSS property in Definition 2.1 from the state to the estimation error, as shown below.

Lemma 2.1: For the closed-loop system given by (1)-(6), there exists a constant $\varsigma$, with $0<\varsigma<\zeta$, such that (7) is equivalent to

$$
\limsup _{k \rightarrow \infty} \mathbb{E}\left[P_{k \mid k}\right] \leq \varsigma .
$$

Proof: The estimate at the controller in (5) and the state can be rewritten as

$$
\begin{aligned}
\hat{x}_{k \mid k}^{c} & =(a-b L) \hat{x}_{k-1 \mid k-1}^{c}+\delta_{k}\left(a \tilde{x}_{k-1 \mid k-1}^{c}+w_{k-1}\right) \\
x_{k} & =(a-b L) \hat{x}_{k-1 \mid k-1}^{c}+\left(a \tilde{x}_{k-1 \mid k-1}^{c}+w_{k-1}\right) .
\end{aligned}
$$

Since $\hat{x}_{k-1 \mid k-1}^{c}$ is the minimum mean square error estimate [11], we can write $\mathbb{E}\left[\left|x_{k}\right|^{2}\right]$ as

$$
\mathbb{E}\left[\left|x_{k}\right|^{2}\right]=(a-b L)^{2} \mathbb{E}\left[\left|\hat{x}_{k-1 \mid k-1}^{c}\right|^{2}\right]+\mathbb{E}\left[P_{k \mid k}\right],
$$

which must be bounded in steady state for stability, as per Definition 2.1. For the deterministic system, the control law in (6) ensures mean square boundedness of the state in steady state, due to optimality. The Certainty Equivalence Principle holds, and thus, this control law ensures mean square boundedness of the estimate $\hat{x}_{k-1 \mid k-1}^{c}$ in (9). Hence the stability condition depends only on the estimation error, and $x_{k}$ is LMSS iff $\lim \sup _{k \rightarrow \infty} \mathbb{E}\left[P_{k \mid k}\right] \leq \varsigma$.

In the rest of the paper, we identify sufficient conditions that guarantee LMSS, in the sense of (8), for the states of each of the $M$ closed-loop systems described above.

\section{B. Network Interaction Model}

We have defined a model and a notion of stability for each closed-loop system. However, we need to model the interactions in the network of $M$ closed-loop systems, and define a notion of stability for the entire network. To do this, we use a Markov chain to jointly model the state-based scheduler and CRM, through which each closed-loop system interacts with the rest of the network. Such a Markovian representation is possible because the delay since the last

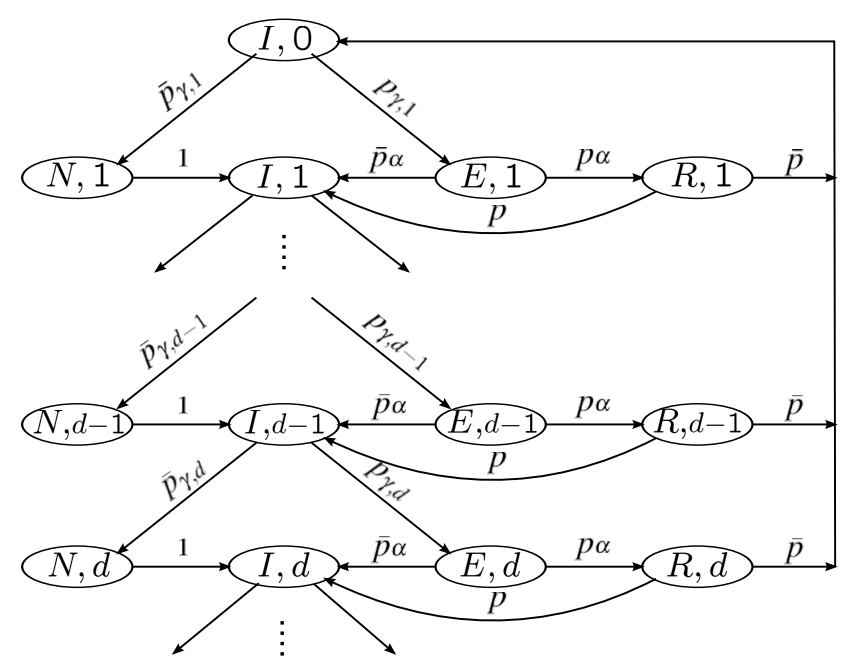

Fig. 4. A Markov chain representation for a state-based scheduler and a simple CRM such as $p$-persistent CSMA with no retransmissions.

successful transmission $d_{k-1}$ is a sufficient statistic for the transmission history $\left\{\delta_{0}, \ldots, \delta_{k-1}\right\}$, for the scheduler in (2).

The delay $d$, along with another index $S$, is used to denote each state in the Markov chain in Fig. 4. To understand this Markov chain, let us trace through the chain for some delay $d_{k}=d-1$, or with the plant in the idle state $(I, d-1)$. At the next sampling instant, the state $x$ is declared to be one of the following.

- Event: The process transitions to $(E, d)$, with probability $p_{\gamma, d}:=\mathbb{P}\left(\gamma_{k}=1 \mid d_{k}=d-1\right)$.

- Non-event: The process transitions to $(N, d)$, with complimentary probability $\bar{p}_{\gamma, d}=1-p_{\gamma, d}$. Then, it transitions directly to the next idle state $(I, d)$, to wait for the next sampling instant.

An event is sent to the CRM, where it is

- Transmitted: The process transitions to $(R, d)$, with probability $p_{\alpha}$.

- Suppressed: With complimentary probability $\bar{p}_{\alpha}$, it returns to the next idle state $(I, d)$.

A transmitting node, in any of the $(R, d)$ states, sees a busy channel if another node in the network is in one of its transmission states, and transmitting during a busy channel causes a collision. Thus, a transmission results in

- Collision: The process transitions, with probability $p$, defined in (10) below, to the idle state $(I, d)$.

- Success: The process transitions, with probability $\bar{p}=$ $1-p$, to the state $(I, 0)$, with the delay reset to $d=0$.

We now present our first assumption, used in the construction of this Markov chain.

Assumption 2.1: The conditional probability of a busy channel, as seen by a node in $(R, d)$, for $d>0$, is assumed to be a time-average, which is independent of the current delays of the interfering nodes, and is given by

$$
p=1-\left(1-\sum_{d=1}^{\infty} p_{(R, d)}\right)^{M-1}
$$

where, $p_{(R, d)}$ is the probability of being in the state $(R, d)$. 
This assumption was first made by Bianchi in his muchacclaimed analysis of CSMA/CA in 802.11 [12], and has been verified with simulations, for the problem setup considered in this paper, in [7]. We can now define a notion of stability for the interactions of the $M$ closed-loop systems in this network.

Definition 2.2 (Network Steady State): The network of $M$ closed-loop systems, given by (1)-(6), is said to be in steady state when the states $(S, d), \forall S \in\{I, N, E, R\}, d \geq$ 0 , are recurrent.

We present the second assumption, which we use in the rest of the analysis.

Assumption 2.2: The network of $M$ closed-loop systems, given by (1)-(6), is assumed to be in steady state.

This assumption implies that $0 \leq p<1$, because if $p=1$, then all the states in this Markov chain are transient, except for the infinite-delay states, and no steady state exists. This, in turn, requires $0 \leq \sum_{d=1}^{\infty} p_{(R, d)}<1$, from (10).

In steady state, we can analyze the performance of any of the closed-loop systems in this network [7]. The probability of a node in the states $(I, d)$ and $(R, d)$ are given by

$$
p_{(I, d)}=\left(1-p_{\gamma, d} p_{\alpha} \bar{p}\right) p_{(I, d-1)}, p_{(R, d)}=p_{\gamma, d} p_{\alpha} p_{(I, d-1)} .
$$

Then, the probability of a successful transmission is given by $\mathbb{P}\left(\delta_{k}=1\right)=p_{(I, 0)}$ and can be obtained by simultaneously solving $\sum_{d=0}^{\infty} p_{(I, d)}=1$ and (10) above.

Thus, we begin by assuming that a steady state exists, and then proceed to find conditions for LMSS. The steady state assumption is not sufficient to guarantee LMSS. However, the LMSS conditions we derive guarantee that Assumption 2.2 holds.

\section{MAIN RESUlts}

We can use the Markov chain in Fig. 4 to analyze stability of each closed-loop system. However, the transition probabilities $p_{\gamma, d}$ in the Markov chain are dependent on the state of the plant, conditioned on its state in the Markov chain itself. Computing these scheduler probabilities, is not a simple task, as explained below. We overcome this difficulty by finding an approximation, which permits us to predict the stability of the system.

\section{A. Probability Distributions for the Markov Chain States}

To use Lemma 2.1, we need to find an expression for the estimation error covariance. Let us associate, with each state $(S, d)$ for $S \in\{I, N, E, R\}$, a probability distribution function (pdf) for the estimation error at the controller, denoted by $\phi_{(S, d)}:=\left\{\phi\left(\tilde{x}_{k \mid k}^{c}\right): \tilde{x}_{k \mid k}^{c} \in(S, d), d_{k}=d\right\}$.

Then, the estimation error covariance conditioned on a delay $d$ is given by $P_{d}=\int_{-\infty}^{\infty} \tilde{x}^{2} \phi_{(I, d)}(\tilde{x}) d \tilde{x}$. Marginalizing over the delay distribution, we get

$$
\mathbb{E}\left[P_{k \mid k}\right]=\sum_{d=0}^{\infty} P_{d} \mathbb{P}\left(d_{k}=d\right) .
$$

This expression is easy to analyze for stability, using the Markov chain in Fig. 4 [13]. However, these pdf(s) can be hard to compute. To see why, let us look at the evolution of these $\operatorname{pdf}(\mathrm{s})$ as the delay $d$ increases. For $d=0$, it is easy to see that $\phi_{(I, 0)}=\phi_{N}\left(\sigma_{w}^{2}\right)$, where $\phi_{N}$ is the pdf of a normal distribution with variance $\sigma_{w}^{2}$. For any delay $d$, the $\operatorname{pdf}(\mathrm{s})$ associated with the event and non-event states are constrained versions of the pdf associated with the previous idle state. Thus, we get

$$
\begin{aligned}
& \phi_{(N, d)}= \begin{cases}\frac{\phi_{(I, d-1)}(\tilde{x})}{\bar{p}_{\gamma, d}} & |\tilde{x}| \leq \epsilon_{d}, \\
0 & \text { otherwise },\end{cases} \\
& \phi_{(E, d)}= \begin{cases}\frac{\phi_{(I, d-1)}(\tilde{x})}{p_{\gamma, d}} & |\tilde{x}|>\epsilon_{d}, \\
0 & \text { otherwise },\end{cases}
\end{aligned}
$$

where, $\bar{p}_{\gamma, d}=\int_{-\epsilon_{d}}^{\epsilon_{d}} \phi_{(I, d-1)}(\tilde{x}) d \tilde{x}$ is the probability of a nonevent and $p_{\gamma, d}=1-\bar{p}_{\gamma, d}$ is the probability of an event after a delay $d$.

Then, let us denote $e_{d}$ as the innovations process which does not get transmitted after a delay $d$. Its pdf is given by

$$
\begin{aligned}
\phi_{(I, d)}^{e}= & \mathbb{P}\left(\tilde{x}_{k \mid k}^{c} \mid \delta_{k}=0, d_{k}=d\right) \\
= & \sum_{\gamma_{k} \in\{0,1\}} \mathbb{P}\left(\tilde{x}_{k \mid k}^{c} \mid \gamma_{k}, \delta_{k}=0, d_{k}=d\right) \cdot \mathbb{P}\left(\gamma_{k} \mid \delta_{k}=0, d_{k}=d\right) \\
= & \phi_{(N, d)}(\tilde{x}) \cdot \frac{\bar{p}_{\gamma, d}}{\bar{p}_{\gamma, d}+p_{\gamma, d}\left(\bar{p}_{\alpha}+p p_{\alpha}\right)} \\
& +\phi_{(E, d)}(\tilde{x}) \cdot \frac{\left(\bar{p}_{\alpha}+p p_{\alpha}\right) p_{\gamma, d}}{\bar{p}_{\gamma, d}+p_{\gamma, d}\left(\bar{p}_{\alpha}+p p_{\alpha}\right)} \\
= & \begin{cases}\phi_{(I, d-1)}(\tilde{x}) \cdot \frac{1}{\bar{p}_{\gamma, d}+p} p_{\gamma, d}\left(\bar{p}_{\alpha}+p p_{\alpha}\right) & |\tilde{x}| \leq \epsilon_{d} \\
\phi_{(I, d-1)}(\tilde{x}) \cdot \frac{\left(\bar{p}_{\alpha}+p p_{\alpha}\right)}{\bar{p}_{\gamma, d}+p_{\gamma, d}\left(\bar{p}_{\alpha}+p p_{\alpha}\right)} & |\tilde{x}|>\epsilon_{d}\end{cases}
\end{aligned}
$$

where, the last expression was obtained by substituting for $\phi_{(N, d)}$ and $\phi_{(E, d)}$ from (13) and (14), respectively. Finally, the pdf of the idle state with delay $d$ is obtained from the plant dynamics in (1) as

$$
\phi_{(I, d)}=\frac{1}{a} \phi_{(I, d)}^{e}\left(\frac{\tilde{x}}{a}\right) * \phi_{N}\left(\sigma_{w}^{2}\right),
$$

which is obtained through a convolution of two $\operatorname{pdf}(s)$.

The above operations must be performed recursively, to obtain the pdf associated with the state $(I, d)$ for all $d>0$, which is hard to compute. Hence, we find approximations for the $\operatorname{pdf}(\mathrm{s})$ and bounds for the estimation error covariance in the following subsections.

\section{B. Two Extreme Network Scenarios}

To begin with, we identify two simple network scenarios, no exogenous network traffic and saturated network traffic.

Lemma 3.1 (Case 1: No exogenous network traffic, $p=0$ ): For the system given by (1)-(6), with no exogenous network traffic $\left(n_{k}=0\right)$, it holds that $P_{k \mid k} \leq \max _{d} \epsilon_{d}^{2}$.

Proof: With no exogenous network traffic, $\delta_{k}=\gamma_{k}$. Thus, we have $\sup _{\left\{\gamma_{0}, \ldots, \gamma_{k}\right\}} P_{k \mid k} \leq \epsilon_{d}^{2}$ for $d=d_{k}$, when $\gamma_{k}=0$, and $P_{k \mid k}=0$ otherwise. Hence, $P_{k \mid k} \leq \max _{d} \epsilon_{d}^{2}$. In this case, all plants achieve LMSS.

Lemma 3.2 (Case 2: Saturated network traffic, $p=1$ ): For the system given by (1)-(6), LMSS is achievable if $\lim _{d \rightarrow \infty} P_{d}$ is bounded. 
Proof: When $p=1, \phi_{(I, d)}^{e}=\phi_{(I, d)}$ as can be seen from (15). Then, $\phi_{(I, d)}=a^{-1} \phi_{(I, d-1)} * \phi_{N}$. Definition 2.2 does not hold, and hence $\lim _{d \rightarrow \infty} p_{(I, d)}=1$.

Thus, stability is determined by the open loop plant. When $a<1$, the estimation error covariance is given by $\mathbb{E}\left[P_{k \mid k}\right]=$ $\frac{\sigma_{w}^{2}}{1-a^{2}}$. Unstable plants cannot be stabilized with saturated network traffic. However, to identify conditions required for stability, we need to find a suitable approximation that can be used even for unstable plants.

\section{Approximation of PDFs using Majorization Theory}

Let us choose $\hat{\phi}_{(S, d)}$ to approximate $\phi_{(S, d)}$ such that $\phi_{(S, d)} \succeq \hat{\phi}_{(S, d)}$. We define the majorization operator $\succeq$ below.

Definition 3.1: The pdf $\phi_{a}$ is said to majorize another pdf $\phi_{b}$, denoted as $\phi_{a} \succeq \phi_{b}$, if

$$
\int_{-L}^{L} \phi_{a}(\ell) d \ell \geq \int_{-L}^{L} \phi_{b}(\ell) d \ell, \forall L \in \mathbb{R} .
$$

This results in an ordering of the second moments, $P_{v}=$ $\int_{v=-\infty}^{\infty}(v-\mathbb{E}[v])^{2} \phi_{v} d v$ for $v=\{a, b\}$, as stated below.

Lemma 3.3: If $\phi_{a} \succeq \phi_{b}$, then $P_{a} \leq P_{b}$.

Proof: $\quad$ Since $\phi_{a} \succeq \phi_{b}$, and $\int_{-\infty}^{\infty} \phi_{a}(\ell) d \ell=$ $\int_{-\infty}^{\infty} \phi_{b}(\ell) d \ell$, there is more probability mass in larger $\ell$ in $\phi_{b}$. Thus, its second moment is larger.

Using Definition 3.1, we find $\hat{\phi}_{(I, d)}$, as stated below.

Theorem 3.4: Let the pdf, $\hat{\phi}_{(I, d)}$, be defined by the recursive relation

$$
\hat{\phi}_{(I, d)}=\frac{1}{a} \hat{\phi}_{(I, d-1)} * \phi_{N},
$$

with $\hat{\phi}_{(I, 0)}=\phi_{N}$. Then, $\phi_{(I, d)} \succeq \hat{\phi}_{(I, d)}$ for all $d \geq 0$.

Proof: We show this using induction. Trivially, at $d=$ $0, \phi_{(I, 0)}=\hat{\phi}_{(I, 0)}=\phi_{N}$. Let us assume that, for some $d$, $\phi_{(I, d)} \succeq \hat{\phi}_{(I, d)}$. Then, from (15), we can show that $\phi_{(I, d+1)}^{e} \succeq$ $\phi_{(I, d)}$. To see this, note that -

- For $|e| \leq \epsilon_{d+1}$, we have

$$
\int_{-e}^{e} \frac{\phi_{(I, d)}(\tilde{x})}{\bar{p}_{\gamma, d+1}+p_{\gamma, d+1}\left(\bar{p}_{\alpha}+p p_{\alpha}\right)} d \tilde{x} \geq \int_{-e}^{e} \phi_{(I, d)}(\tilde{x}) d \tilde{x},
$$

because $\bar{p}_{\gamma, d+1}+p_{\gamma, d+1}\left(\bar{p}_{\alpha}+p p_{\alpha}\right) \leq 1$.

- For $|e|>\epsilon_{d+1}$, we have

$$
\begin{aligned}
\int_{-\infty}^{-e} & \phi_{(I, d)}(\tilde{x}) \frac{\left(\bar{p}_{\alpha}+p p_{\alpha}\right)}{\bar{p}_{\gamma, d+1}+p_{\gamma, d+1}\left(\bar{p}_{\alpha}+p p_{\alpha}\right)} d \tilde{x} \\
& +\int_{e}^{\infty} \phi_{(I, d)}(\tilde{x}) \frac{\left(\bar{p}_{\alpha}+p p_{\alpha}\right)}{\bar{p}_{\gamma, d+1}+p_{\gamma, d+1}\left(\bar{p}_{\alpha}+p p_{\alpha}\right)} d \tilde{x} \\
& \leq \int_{-\infty}^{-e} \phi_{(I, d)}(\tilde{x}) d \tilde{x}+\int_{e}^{\infty} \phi_{(I, d)}(\tilde{x}) d \tilde{x},
\end{aligned}
$$

because $\frac{\left(\bar{p}_{\alpha}+p p_{\alpha}\right)}{\bar{p}_{\gamma, d+1}+p_{\gamma, d+1}\left(\bar{p}_{\alpha}+p p_{\alpha}\right)} \leq 1$.

Since $\phi_{(I, d+1)}^{e} \succeq \phi_{(I, d)}$ and $\phi_{(I, d)} \succeq \hat{\phi}_{(I, d)}$, we have

$$
\begin{aligned}
\phi_{(I, d+1)}^{e} & \succeq \hat{\phi}_{(I, d)} \\
\frac{1}{a} \phi_{(I, d+1)}^{e}\left(\frac{\tilde{x}}{a}\right) & \succeq \frac{1}{a} \hat{\phi}_{(I, d)}\left(\frac{\tilde{x}}{a}\right) \\
\phi_{N} * \frac{1}{a} \phi_{(I, d+1)}^{e}\left(\frac{\tilde{x}}{a}\right) & \succeq \phi_{N} * \frac{1}{a} \hat{\phi}_{(I, d)}\left(\frac{\tilde{x}}{a}\right),
\end{aligned}
$$

where, the above relationships follow from the properties of the majorization operator for neat distributions [14]. Hence, $\phi_{(I, d+1)} \succeq \hat{\phi}_{(I, d+1)}$, and, this is true for all $d \geq 0$.

The pdf(s) given by $\hat{\phi}_{(I, d)}$ define the worst-case evolution of the system considered in Lemma 3.2, where $p=1$.

\section{Stability Conditions for the Markov Chain}

Let us now look at stability conditions for the Markov chain in Fig. 4.

Lemma 3.5: For the closed-loop system given by (1)-(6), under Assumption 2.2, sufficient conditions for LMSS are given by

$$
\begin{aligned}
\limsup _{d \rightarrow \infty} \frac{p_{(I, d+1)}}{p_{(I, d)}} & <\frac{1}{1+a^{2}} \\
\sum_{d=1}^{\infty} \frac{p_{\gamma, d}}{1+a^{2}} & <\frac{1}{p_{\alpha} p_{(I, 0)}}
\end{aligned}
$$

Proof: The estimation error covariance can be bounded from above, using the approximations from Theorem 3.4, as

$$
\mathbb{E}\left[P_{k \mid k}\right]=\sum_{d=1}^{\infty} p_{(I, d)} P_{d} \leq \sum_{d=1}^{\infty} p_{(I, d)} \hat{P}_{d}
$$

For this expression to be bounded [15], we require

$$
\limsup _{d \rightarrow \infty} \frac{p_{(I, d+1)} \hat{P}_{d+1}}{p_{(I, d)} \hat{P}_{d}}<1 .
$$

Since $\hat{P}_{d}=\sigma_{w}^{2}\left(1+a^{2}+\cdots+a^{2 d}\right)$, the left hand side of the above inequality can be written as

$$
\begin{aligned}
& \limsup _{d \rightarrow \infty} \frac{p_{(I, d+1)}}{p_{(I, d)}}\left[1+a^{2} \frac{a^{2 d}}{1+a^{2}+\cdots+a^{2 d}}\right] \\
\leq & \limsup _{d \rightarrow \infty} \frac{p_{(I, d+1)}}{p_{(I, d)}}\left[1+a^{2}\right] .
\end{aligned}
$$

By requiring the last expression to be strictly less than 1 in (16), we satisfy the conditions required to obtain LMSS

The above analysis is only possible in steady state (Assumption 2.2), which requires that $\sum_{d=1}^{\infty} p_{(R, d)}<1$. Substituting for $p_{(R, d)}$ from (11) in this expression, and using (16), we obtain (17).

Let us assume that we choose the scheduler probabilities such that

$$
p_{(I, d+1)}<\frac{p_{(I, d)}}{1+a^{2}},
$$

and such that (16) is true. Then, using this in $\sum_{d=0}^{\infty} p_{(I, d)}=$ 1 , we get $p_{(I, 0)}>a^{2} /\left(1+a^{2}\right)$.

Finally, we derive some conditions to achieve the requirements in Lemma 3.5, and hence LMSS.

Theorem 3.6: For the closed-loop system given by (1)(6), under Assumption 2.2, sufficient conditions for LMSS are given by

$$
\begin{aligned}
\limsup _{d \rightarrow \infty} p_{\gamma, d} & >\kappa_{\alpha} \frac{1}{\left(1-p_{c}\right)^{M-1}}, \\
\sum_{d=1}^{\infty} \frac{p_{\gamma, d}}{\left(1+a^{2}\right)^{d-1}} & <\frac{1}{p_{\alpha}}\left(1-\kappa_{\alpha}^{1 /(M-1)}\right) \frac{1}{p_{(I, 0)}},
\end{aligned}
$$

where, $p_{c}=p_{\alpha} p_{(I, 0)} \sum_{\ell=1}^{\infty} \frac{p_{\gamma, \ell}}{\left(1+a^{2}\right)^{\ell-1}}$ and $\kappa_{\alpha}=\frac{1}{p_{\alpha}} \frac{a^{2}}{1+a^{2}}$. 
Proof: From the Markov chain in Fig. 4, we can use the recursive relationship for the pdf of the state $(I, d+1)$ in (11) along with the sufficient condition in (16) to obtain

$$
\begin{aligned}
\limsup _{d \rightarrow \infty} \frac{\left(1-p_{\gamma, d+1} p_{\alpha} \bar{p}\right) p_{(I, d)}}{p_{(I, d)}} & <\frac{1}{1+a^{2}} \\
\limsup _{d \rightarrow \infty} p_{\gamma, d+1} \bar{p} & >\frac{1}{p_{\alpha}} \frac{a^{2}}{1+a^{2}}=\kappa_{\alpha} .
\end{aligned}
$$

Substituting for $\bar{p}$ from (10) and the sufficient condition in (18), we get

$$
\begin{aligned}
& \limsup _{d \rightarrow \infty} p_{\gamma, d+1}\left(1-\sum_{\ell=1}^{\infty} p_{\gamma, \ell} p_{\alpha} p_{(I, \ell-1)}\right)^{M-1} \\
& \geq \limsup _{d \rightarrow \infty} p_{\gamma, d+1}\left(1-p_{\alpha} p_{(I, 0)} \sum_{\ell=1}^{\infty} \frac{p_{\gamma, \ell}}{\left(1+a^{2}\right)^{\ell-1}}\right)^{M-1}
\end{aligned}
$$

Using the definition of $p_{c}$ and setting the above expression to be greater than $\kappa_{\alpha}$ to achieve (21), we obtain the bound in (19).

For $0<p_{\gamma, d}<1$, the above inequality results in

$$
\kappa_{\alpha} \frac{1}{\left(1-p_{c}\right)^{M-1}}<1
$$

which can be rearranged to obtain (20).

Steady State versus LMSS: As mentioned earlier, Assumption 2.2 is not sufficient to guarantee LMSS. This can be seen by noting that a steady state requires $p<1$. From (11), this implies that $p_{(I, d+1)}<p_{(I, d)}$. Thus, the network steady state ensures that the loop is sometimes closed, as against the case $p=1$, when the loop is never closed. But, this feedback may not be sufficient to stabilize the system, in the sense of Definition 2.1.

However, LMSS for all $M$ systems in the network ensures a network steady state, in the sense of Definition 2.2. To see this, note that the upper bound in (20) ensures that (17) is satisfied. This is because $\kappa_{\alpha}<1$, from (21). Thus, in (20), $\left(1-\kappa_{\alpha}^{(1 /(M-1))}\right)<1$. Hence, a steady state is indeed achieved by the closed-loop systems in stability.

\section{DESIGN OF THE SCHEDULER}

Now that we have some constraints on the scheduling probabilities, what does this mean for the design of the scheduler itself? In particular, how should $p_{\gamma, d}$ be designed as a function of $d$ to achieve LMSS? We can immediately think of three possible ways to vary the scheduling probability with delay: holding it a constant, additively increasing or decreasing it. We do not consider multiplicatively increasing or decreasing the scheduler probabilities as it is easy to show that this would negate the conditions of Theorem 3.6. We discuss each of the other designs, and identify stability conditions for these schedulers.

\section{A. Constant Scheduling Laws}

This scheduler is designed to provide a constant scheduling probability for all delays, ie. $p_{\gamma, d}=p_{\gamma}$, for all $d>0$. Applying the constraints obtained from Theorem 3.6, we identify stability conditions for this particular law.
Corollary 4.1: For the closed-loop system given by (1)(6), a sufficient condition for LMSS for constant scheduling laws is given by

$$
\kappa_{\alpha} \frac{1}{\left(1-p_{c}\right)^{M-1}}<p_{\gamma}<\kappa_{\alpha}\left(1-\kappa_{\alpha}^{1 /(M-1)}\right) \frac{1}{p_{(I, 0)}} .
$$

Proof: The lower bound on $p_{\gamma}$ comes from the constraint in (19). To obtain the upper bound, note that the left hand side of (20) can be evaluated as $p_{\gamma} \frac{1+a^{2}}{a^{2}}$. Substituting in (20) and rearranging, gives us (23).

\section{B. Additive Scheduling Laws}

This scheduler is designed to provide an additive increase/decrease in the scheduling probability with delay, i.e., $p_{\gamma, d}=p_{\gamma, d-1}+\nu$, for $\nu \gtrless 0$. Since $\max p_{\gamma, d}<1$, this scheduling law can only be defined when the additive terms decrease in magnitude. A necessary condition is obtained by requiring that the sum of the series in (20) is summable and is given by $\lim \sup _{d \rightarrow \infty} p_{\gamma, d+1} / p_{\gamma, d}<1+a^{2}$. We look at one of many possible examples, for increasing and decreasing scheduling probabilities, to see the effects on the bounds.

Let us first consider an increasing sequence of scheduler probabilities, defined by

$$
p_{\gamma, d}=p_{\gamma, 1}+\eta+\eta^{2}+\cdots+\eta^{d-1}, \quad \text { for } \eta<1 .
$$

Thus, $\lim _{d \rightarrow \infty} p_{\gamma, d}=p_{\gamma, 1}+\frac{\eta}{1-\eta}$, and $p_{\gamma, 1}$ and $\eta$ must be chosen such that this term is less than 1 . Then, we have the following result.

Corollary 4.2: For the closed-loop system given by (1)(6), a sufficient condition for LMSS for the additive and increasing scheduling law in (24), is given by

$$
\kappa_{\alpha} \frac{1}{\left(1-p_{c}\right)^{M-1}}<p_{\gamma, \max }<\kappa_{\alpha}\left(1-\kappa_{\alpha}^{1 /(M-1)}\right) \frac{1}{p_{(I, 0)}}+\kappa_{\eta},
$$

where, $p_{\gamma, \text { max }}=p_{\gamma, 1}+\frac{\eta}{1-\eta}$ and $\kappa_{\eta}=\frac{\eta}{1-\eta} \cdot \frac{a^{2}}{1+a^{2}-\eta}$.

Proof: The maximum scheduling probability is given by $p_{\gamma, \max }=\lim _{d \rightarrow \infty} p_{\gamma, d}$, and thus the lower bound on $p_{\gamma, \max }$ comes from the constraint in (19). The upper bound is obtained by evaluating the left hand side in (20) and rearranging the resulting condition.

Now, let's consider a decreasing sequence of scheduler probabilities, defined by

$$
p_{\gamma, d}=p_{\gamma, 1}-\beta-\beta^{2}-\cdots-\beta^{d-1}, \quad \text { for } \beta<1 .
$$

Thus, $\lim _{d \rightarrow \infty} p_{\gamma, d}=p_{\gamma, 1}-\frac{\beta}{1-\beta}$, and $p_{\gamma, 1}$ and $\beta$ must be chosen such that this term is less than the bound in (19), and $p_{\gamma, 1}<1$. Then, we have the following result.

Corollary 4.3: For the closed-loop system given by (1)(6), a sufficient condition for LMSS for the additive, but decreasing, scheduling law in (25), is given by

$$
\begin{aligned}
\kappa_{\alpha} \frac{1}{\left(1-p_{c}\right)^{M-1}} & +\frac{\beta}{1-\beta}<p_{\gamma, 1} \\
& <\kappa_{\alpha}\left(1-\kappa_{\alpha}^{1 /(M-1)}\right) \frac{1}{p_{(I, 0)}}+\frac{\beta}{1+a^{2}-\beta} .
\end{aligned}
$$

The proof is identical to the one for Corollary 4.3 , with the exception that $\max p_{\gamma, d}=p_{\gamma, 1}$. 


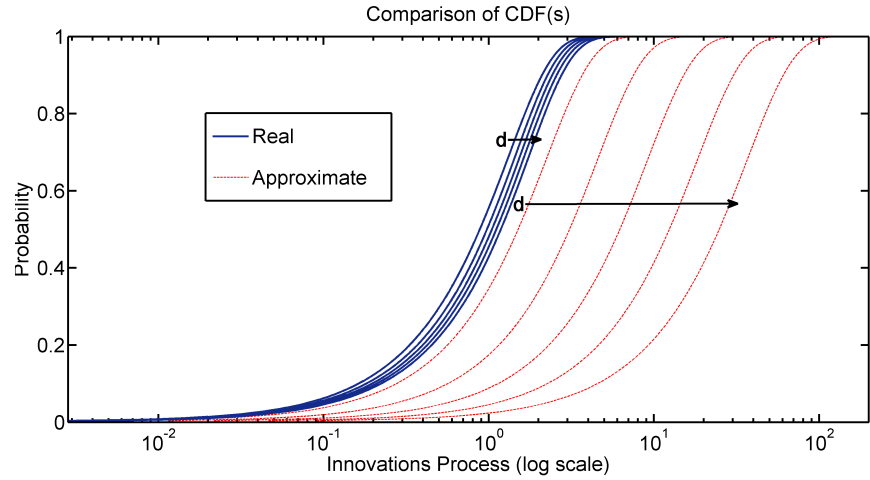

Fig. 5. The approximate pdf in Theorem 3.4 is majorized by the actual pdf, as seen in this comparison of the $\operatorname{cdf}(\mathrm{s})$. The approximate distribution has a larger variance, and is an upper bound for the actual variance.

\section{EXAMPLE}

We now illustrate some of the results presented above. In Fig. 5, we illustrate that $\hat{\phi}_{(I, d)}$ from Theorem 3.4 is majorized by $\phi_{(I, d)}$ for $d=\{1, \ldots, 5\}$, by comparing their cumulative distribution functions $(\operatorname{cdf}(\mathrm{s}))$. The arrow indicates the variation in the $\operatorname{cdf}(\mathrm{s})$, with increasing delay. We use a closed-loop system with parameters $a=2, b=1$, $\sigma_{w}=1$ and $\epsilon_{d}=1$, for all $d>0$. The CRM persistence probability is set to $p_{\alpha}=1$, and the conditional probability of a busy channel is $p=0.6$. Note that the approximation depends on the dynamics of the plant, and it was chosen to correspond to the worst-case evolution of the real $\mathrm{pdf}(\mathrm{s})$, with saturated network traffic. Thus, the approximation is tighter for large $p$ and small $a$.

Next, we consider two network scenarios: case 1 corresponds to a network with $M=2$ nodes, and case 2 with $M=10$ nodes. The rest of the parameters are $a=1, b=1$, $\sigma_{w}=1$ and $\epsilon_{d}=0.25, \forall d>0$. The CRM presented in this paper permits no retransmissions, and cannot support many unstable processes. Our analysis shows this; By applying the constant law design from Corollary 4.1, we do not find any set of parameters $p_{\gamma}, p_{c}$ and $p_{I, 0}$ that guarantee LMSS.

However, the Markov analysis extends to a CRM with retransmissions [7]. For 10 retransmissions, we use the results of Lemma 3.5, which apply here as well, to show that for case 1, LMSS is achievable, and for case 2, LMSS cannot be guaranteed. Using (16), we see that the idle state probabilities must achieve a ratio of less than 0.5 , for large $d$. Case 1 achieves a ratio of less than 0.1 for $d>10$, whereas case 2 has a ratio of 0.98 even after $d=50$. The LMSS properties can be inferred from a trace of the state $x$, for each case, in Fig. 6.

\section{Conclusions}

We have derived a set of sufficient conditions on the probability of delay since the last transmission that ensure LMSS of the event-based NCS, with state-based schedulers and an explicit CRM. We have translated these conditions to constraints on the scheduling probabilities. Using these constraints, we have identified three scheduling laws, along with sufficient conditions to obtain LMSS. Future work

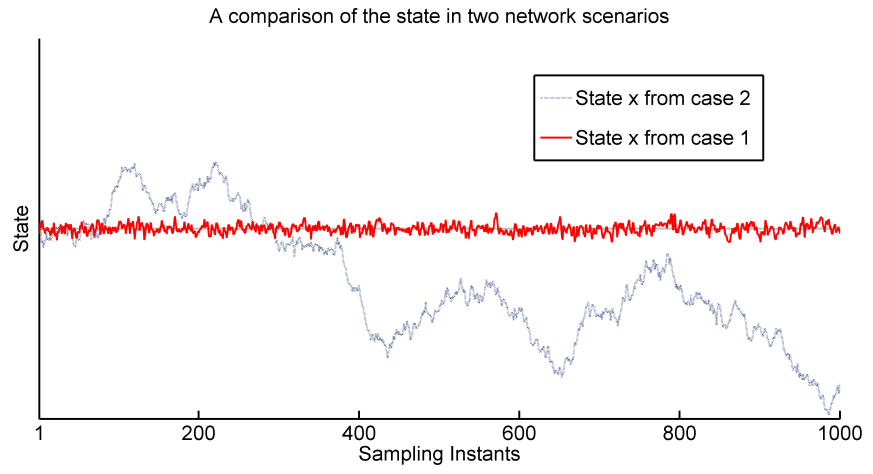

Fig. 6. A comparison of the trace of the plant state $x$ for two different network scenarios: case 1 is guaranteed to be LMSS by the results of Lemma 3.5, while case 2 is not.

includes extending the laws to a CRM with retransmissions and translating scheduling laws into policies for modifying the scheduler thresholds.

\section{REFERENCES}

[1] P. G. Otanez, J. R. Moyne, and D. M. Tilbury, "Using deadbands to reduce communication in networked control systems," in Proceedings of the American Control Conference, vol. 4, pp. 3015 - 3020 vol.4, 2002.

[2] K. J. Åström and B. M. Bernhardsson, "Comparison of Riemann and Lebesgue sampling for first order stochastic systems," in Proceedings of the 41st IEEE Conference on Decision and Control, vol. 2, pp. 2011 -2016 vol.2, 2002.

[3] L. Kleinrock and F. Tobagi, "Packet switching in radio channels: Part I-carrier sense multiple-access modes and their throughputdelay characteristics," IEEE Transactions on Communications, vol. 23, pp. 1400 - 1416, Dec. 1975.

[4] M. Rabi and K. H. Johansson, "Scheduling packets for event-triggered control," Proceedings of 10th European Control Conference, pp. 3779 - 3784, 2009.

[5] D. Antunes, J. Hespanha, and C. Silvestre, "Volterra Integral Approach to Impulsive Renewal Systems: Application to Networked Control," IEEE Transactions on Automatic Control, no. 99, pp. 1-1, 2011.

[6] M. Lemmon and X. S. Hu, "Almost sure stability of networked control systems under exponentially bounded bursts of dropouts," in Proceedings of the 14th international conference on Hybrid systems: computation and control - HSCC '11, (New York, New York, USA), p. 301, ACM Press, Apr. 2011.

[7] C. Ramesh, H. Sandberg, and K. H. Johansson, "Steady State Performance Analysis of Multiple State-based Schedulers with CSMA," in Preprints of the 50th IEEE Conference on Decision and Control and European Control Conference, pp. 4729-4734, 2011.

[8] T. Henningsson and A. Cervin, "A simple model for the interference between event-based control loops using a shared medium," in Proceedings of the 49th IEEE Conference on Decision and Control, 2010.

[9] V. Gupta and N. Martins, "On Stability in the Presence of Analog Erasure Channel Between the Controller and the Actuator," IEEE Transactions on Automatic Control, vol. 55, pp. 175-179, Jan. 2010.

[10] F. Kozin, "A survey of stability of stochastic systems," Automatica, vol. 5, no. 1, pp. $95-112,1969$.

[11] C. Ramesh, Contention-based Multiple Access Architectures for Networked Control Systems. Licentiate thesis, KTH Royal Institute of Technology, 2011.

[12] G. Bianchi, "Performance analysis of the IEEE 802.11 distributed coordination function," IEEE Journal on Selected Areas in Communications, vol. 18, pp. 535 -547, Mar. 2000.

[13] S. P. Meyn and R. L. Tweedie, Markov Chains and Stochastic Stability. Springer-Verlag, London, 1993.

[14] B. Hajek, K. Mitzel, and S. Yang, "Paging and Registration in Cellular Networks: Jointly Optimal Policies and an Iterative Algorithm,' IEEE Transactions on Information Theory, vol. 54, pp. 608-622, Feb. 2008.

[15] W. Rudin, Principles of Mathematical Analysis. McGraw-Hill, Inc., third ed., 1976. 